\title{
M.
}

\section{Association between HLA-C*04 and American cutaneous leishmaniasis in endemic region of southern Brazil}

\author{
R.C. Ribas-Silva ${ }^{1}$, A.D. Ribas ${ }^{1}$, E.C. Ferreira ${ }^{1}$, T.G.V. Silveira ${ }^{2}$ and S.D. Borelli ${ }^{3}$ \\ ${ }^{1}$ Programa de Pós-Graduação em Ciências da Saúde, \\ Universidade Estadual de Maringá, Maringá, PR, Brasil \\ 2Laboratório de Leishmanioses, Departamento de Análises Clínicas e Biomedicina, \\ Universidade Estadual de Maringá, Maringá, PR, Brasil \\ ${ }^{3}$ Laboratório de Imunogenética, Departamento de Ciências Básicas da Saúde, \\ Universidade Estadual de Maringá, Maringá, PR, Brasil \\ Correspondence author: R.C. Ribas-Silva \\ E-mail: coord.biomedicina@grupointegrado.br \\ Genet. Mol. Res. 14 (4): 14929-14935 (2015) \\ Received May 12, 2015 \\ Accepted June 16, 2015 \\ Published November 18, 2015 \\ DOI http://dx.doi.org/10.4238/2015.November.18.58
}

\begin{abstract}
Leishmaniasis is a parasitic infectious disease with global repercussions. American cutaneous leishmaniasis $(A C L)$ is endemic in southern Brazil and its pathogenesis varies according to parasite species, immune response, and host genetics. In terms of immunogenetics, many host genes, including HLA (human leukocyte antigen), could be involved in susceptibility to and protection against ACL. Accordingly, the aim of this study was to investigate the association between HLA class I genes (HLA-A, -B, and -C) and ACL in an endemic region of southern Brazil. The allele frequencies of 186 patients diagnosed with $A C L$ and 278 healthy individuals were compared. HLA class I (HLA-A, -B, and -C) typing was carried out by PCR-SSO using Luminex technology. The results revealed an association between the HLA-C ${ }^{*} 04$ allele and the patient study group, in which it appeared more frequently than in the control group [21.5 vs $13.49 \%(P=0.0016$ and $\mathrm{Pc}=0.0258 ; \mathrm{OR}=1.7560 ; 95 \% \mathrm{Cl}=1.2227-$
\end{abstract}


2.5240)], thereby suggesting an increased susceptibility to ACL. Additional allelic groups such as HLA-A*02, HLA-B*35, HLA-B*45, HLA-C*01, and HLA-C*15 were also implicated; however, further investigation is necessary to confirm their association with ACL. Therefore, the results obtained in this study demonstrate the involvement of HLA class I genes in the susceptibility or resistance to $\mathrm{ACL}$, with significant association between HLA-C*04 and ACL susceptibility.

Key words: Genetic susceptibility; HLA; Leishmaniasis

\section{INTRODUCTION}

Leishmaniasis is a parasitic infectious disease caused by protozoa of the genus Leishmania, which are transmitted through the bite of infected sand flies. Depending on the species of Leishmania, the immunological status, and genetic make up of the host, the infection can result in four different clinical manifestations: cutaneous leishmaniasis $(C L)$, diffuse $C L$, mucocutaneous leishmaniasis (MCL), and visceral leishmaniasis (Rey, 2008; Condino et al., 2008; Brasil, 2015).

In Brazil, cases of American cutaneous leishmaniasis (ACL) have been reported since the 1980s. Between 1990 and 2013, 635,399 cases of ACL were reported, of which 13,889 occurred in southern Brazil and of those 13,188 (94.9\%) occurred in the State of Paraná (Brasil, 2015). The northern and western regions of the State of Paraná are considered to be of epidemiological importance due to the largest number of cases occurring in those areas (Lima et al., 2002). In southern Brazil, the predominant species Leishmania (Viannia) braziliensis $(98.7 \%)$ is responsible for the occurrence of the two main forms of leishmaniasis, CL and MCL (Lonardoni et al., 1993; Silveira et al., 1999).

The pathogenesis, clinical patterns, and life cycle of the Leishmania parasite have already been well characterized. As such, immunological studies have shown that when activated Thelper (Th) cells, such as Th2, are dominant, production of interleukin (IL)-4, IL-10, and other cytokines are promoted and render the individual more susceptible and unable to control the infection (Romagnani, 1991; Rogers et al., 2002). Alternatively, when Th1 cells prevail, cytokine IFN-y is secreted, and the individual becomes resistant to infection (Rogers et al., 2002; Murray et al., 2005). However, larger immunogenetic studies such as investigations related to the genetic markers of major histocompatibility complex $(\mathrm{MHC})$ are still required to better understand the reason for different clinical outcomes among immunocompetent hosts infected by the same parasite species.

$\mathrm{MHC}$ is the set of genes responsible for encoding histocompatibility molecules, which in humans is known as human leukocyte antigen (HLA) (Ellis and Ballingall, 1999). HLA is closely linked to the activation of the immune response, as it encodes antigen-presenting proteins to alert immune cells. As such, HLA class I and II alleles have been associated with susceptibility, protection, and clinical manifestations of various diseases including infection by Leishmania spp (Barbier et al., 1987; Lara et al., 1991; Petzl-Erler et al., 1991; Probst et al., 2000; Alves et al., 2006; Sakthianandeswaren et al., 2009). However, these sorts of genetic studies are uncommon in southern Brazil, where to date, only one small study has been conducted to assess a few genetic markers (Petzl-Erler et al., 1991).

Therefore, the aim of this study was to investigate the possible involvement of HLA class 
I genes (HLA-A, -B, -C) in the susceptibility to and protection from ACL among individuals from an endemic region of southern Brazil.

\section{MATERIAL AND METHODS}

\section{Patients and controls}

A retrospective study was conducted using epidemiological records of patients, from the 13th and 15th Regional Health District of the State of Paraná, who were screened and diagnosed with ACL in Laboratório de Leishmanioses at Universidade Estadual de Maringá. To proceed with this investigation, we selected patients that tested positive for the parasite and/or Montenegro skin test.

The study population was formed by a group of 186 patients who were positively diagnosed with ACL and who received prescription of treatment. The control group was composed of 278 healthy individuals who were matched according to age, gender, ethnicity, occupation, and other demographic characteristics, and who presented with no clinical manifestations of the disease and were living in an ACL endemic area. Individuals belonging to the two study groups had no family relation.

During the time of data collection, the patients ranged in age from 17 to 83 years old (mean of $47.06 \pm 14.52$ years), and they comprised $139(82.3 \%)$ males and $30(17.7 \%)$ females. Regarding ethnicity of the patients, 140 (75.3\%) were Caucasian, 40 (21.5\%) Amerindian, 4 (2.2\%) African, and 2 (1.0\%) Asian.

The control/healthy individuals ranged in age from 18 to 73 years old (mean of $33.5 \pm$ 11.28), and they comprised $222(79.9 \%)$ males and 56 (20.1\%) females. The ethnic distribution consisted of 166 (59.7\%) Caucasian, 28 (10.1\%) Amerindian, 11 (4.0\%) African, and 8 (2.9\%) Asian, and 65 chose not to state ethnicity (23.3\%).

The individuals involved in this study were interviewed, and data were collected and recorded in socio-epidemiological reports, which included information concerning clinical manifestations presented by the patient after treatment, such as development of the mucosal form of the disease, or even disease recurrence. All patients were informed about the study to be conducted and were required to sign a consent form upon agreement with participation. This study was approved by the Standing Committee on Ethics in Research Involving Human Beings at Universidade Estadual de Maringá, according to protocol No. 153/2009.

\section{Determination of HLA alleles}

Samples of peripheral blood $(10 \mathrm{~mL})$ were collected from all participants (patients and controls) in tubes containing the anticoagulant EDTA, and centrifuged at $960 \mathrm{~g}$ for $10 \mathrm{~min}$. Nucleated cells were separated and frozen at $-80^{\circ} \mathrm{C}$ until further analysis. Genomic DNA was extracted from $100 \mu \mathrm{L}$ frozen cells using the EZ-DNA extraction kit (Biological Industries, Kibbutz Beit Haemek, Israel) following the manufacturer instructions. The purity and concentration of DNA was determined using a NanoDrop 2000c/2000 UV-Vis spectrophotometer.

HLA class I genotypes (A, B, and C) of patients and controls were analyzed by PCRSSO (polymerase chain reaction-sequence specific oligonucleotide) with Luminex technology (One Lambda Inc., Canoga Park, CA, USA) in the Laboratory of Immunogenetics at Universidade Estadual de Maringá. 


\section{Statistical analysis}

HLA-A, -B and -C genotyping results were tabulated using a database created in Excel 2007. The results were expressed as allele frequency (fa\%), calculated by dividing the number of times different alleles appeared in the sample by the total number of alleles. The $P$ value was calculated by the two-tailed Fisher exact test, and the adjusted $P$ value was calculated using Bonferroni correction adjusted for each locus for multiple comparisons. The odds ratio (OR) was calculated with a $95 \%$ confidence interval $(\mathrm{Cl})$ for $\mathrm{P}$ values $<0.05$. Descriptive statistical analysis for gender and ethnicity variables was carried out using the Fisher exact test, whereas the age variable was calculated by the $t$-test for independent samples. Statistical analyses for all tests were performed using the R program set at a significance level of $5 \%$. Hardy-Weinberg equilibrium (HWE) was determined using the Arlequin v. 2000 program (http://anthropologie.unige.ch/arlequin/).

\section{RESULTS}

In this study, a significant difference was observed between patients with ACL and healthy individuals in regard to the presence of the HLA-C*04 allele. This allele appeared with greater frequency in patients $[21.5$ vs $13.5 \%(P=0.0016$ and $\mathrm{PC}=0.0258 ; \mathrm{OR}=1.7560 ; 95 \% \mathrm{Cl}=1.2227$ 2.5240)], thereby suggesting an increased susceptibility to ACL as compared to healthy individuals (Table 1). Also noteworthy was that HLA-C*04,04 (homozygous) patients were more susceptible to $\mathrm{ACL}$ than heterozygous patients $(\mathrm{P}=0.0206 ; \mathrm{OR}=3.4227 ; 95 \% \mathrm{Cl}=1.0740-12.7812)$.

The possible involvement of ACL and HLA class I was also observed for the following allelic groups: HLA-A ${ }^{*} 02[27.0$ vs 34.0\% $(\mathrm{P}=0.0301$ and $\mathrm{PC}=0.4405 ; \mathrm{OR}=0.7239 ; 95 \% \mathrm{Cl}=$ $0.5361-0.9743)$ ], HLA-B 35 [16.4 vs 10.6\% ( $\mathrm{P}=0.0124$ and $\mathrm{Pc}=0.3118 ; \mathrm{OR}=1.6512 ; 95 \% \mathrm{Cl}=$ 1.1028-2.4744)], HLA-B*45 [0.0 vs $1.8 \%(P=0.0073$ and $\mathrm{Pc}=0.1973)$ ], HLA-C*01 [5.1 vs $2.2 \%$ $(\mathrm{P}=0.0237$ and $\mathrm{Pc}=0.3185 ; \mathrm{OR}=2.4376 ; 95 \% \mathrm{Cl}=1.1076-5.5792)], \mathrm{HLA}-\mathrm{C}^{*} 15[2.7$ vs $6.7 \%$ $(P=0.0087$ and $P c=0.1305 ; O R=0.3878 ; 95 \% C l=0.1697-0.8081)]$. Although these values were significant by the Fisher exact test, no statistical significance was observed upon Bonferroni correction (Table 1). Among these allelic groups, evidence was found in the homozygous analysis of HLA-B* 35,35 , in which 7 individuals were identified in the patient study group compared to only 1 in the control group $(\mathrm{P}=0.0081 ; \mathrm{OR}=10.784 ; 95 \% \mathrm{Cl}=1.3671-4.8875)$.

\section{DISCUSSION}

To investigate whether genetic factors within the $\mathrm{MHC}$ gene cluster region may contribute to susceptibility and/or protection against ACL, the allele frequencies of HLA class I were compared between two groups: ACL-positive patients and healthy individuals. The values obtained for the HLA-C*04 allele $(P=0.0016)$ were significant, with the greatest allele frequency occurring in the patient study group (21.5 vs $13.5 \%$ ). These results suggest a possible involvement of HLA-C*04 in ACL susceptibility.

Lara et al. (1991) previously reported the involvement of the HLA-Cw4 allele $(P=0.0095)$ in ACL in a serological study conducted in family members in Venezuela. Our findings also support the association of $\mathrm{ACL}$ with the $\mathrm{HLA}-\mathrm{C}^{*} 04$ allelic group and to further emphasize this association, significance $(\mathrm{PC}=0.0258)$ was determined by Bonferroni's correction. Findings in the literature also report a correlation of the HLA-C (HLA-Cw7) locus in the pathogenesis of cutaneous leishmaniasis (Barbier et al., 1987; Lara et al., 1991). 


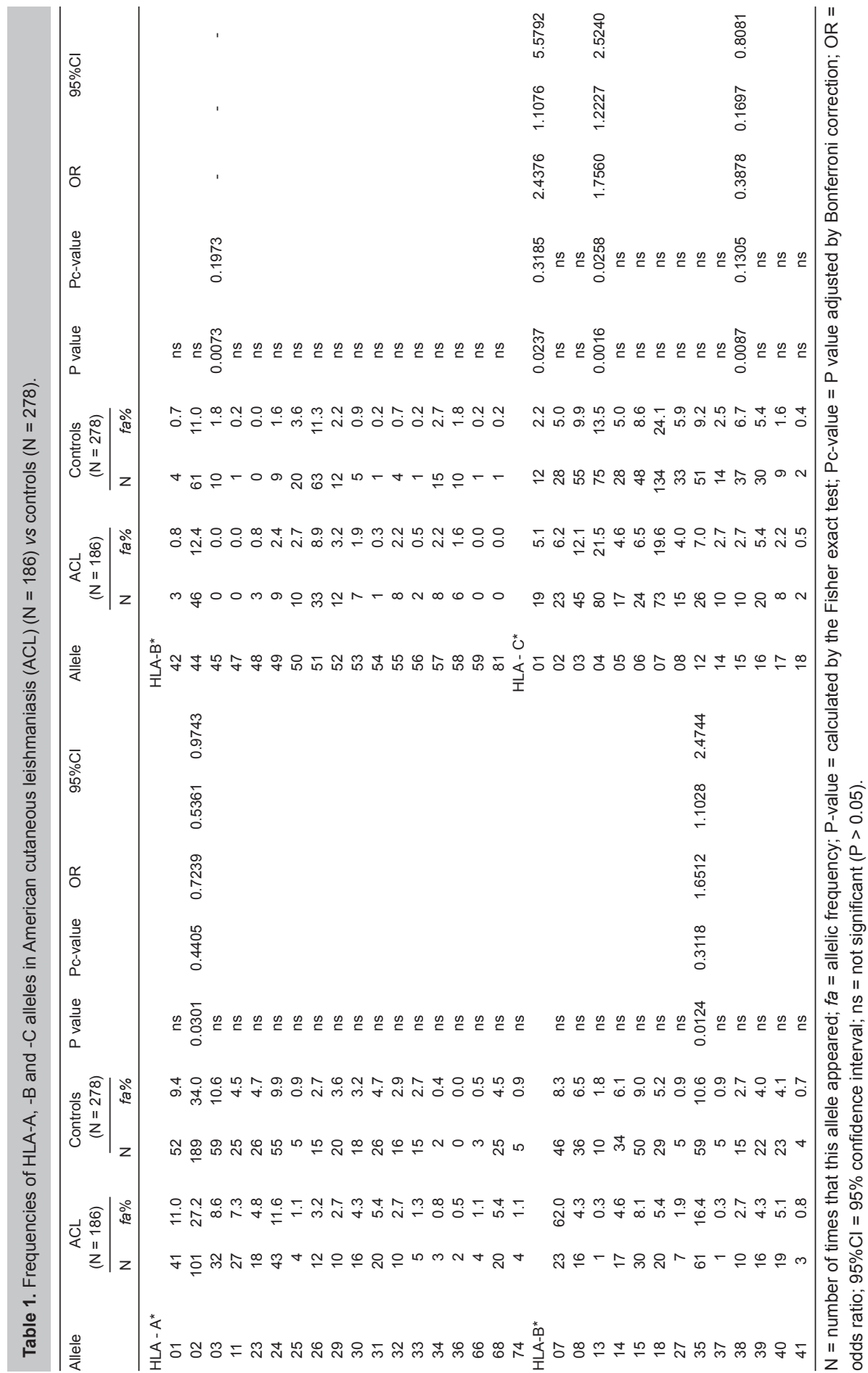


Furthermore in this study, allelic groups HLA-A*02, HLA-B*45, and HLA-C*15 were also considered potential factors of resistance to $A C L$. Moreover, significant $P$ values determined by the Fisher exact test suggest possible involvement in disease susceptibility for HLA-B*35 and HLA-C*01. However, additional studies are required to confirm the conclusion drawn from these results. It is important to note that although in this study, the $P$ value for the HLA-B*45 allelic group $\left(P=0.0073 ; P_{C}=0.1973\right)$ was not significant after Bonferroni's correction, its involvement in $\mathrm{ACL}$ should still be considered because this allele is not very common in the Brazilian population (Allele Frequencies, 2015). In addition, we reported a tendency toward ACL resistance for HLA-B*45 ( $\mathrm{P}=0.0046$; $\mathrm{PC}=0.1426)$ in a previous study conducted with individuals from the same geographic region, compared to a different control group (Petzl-Erler et al., 1991). Furthermore, it is possible to affirm that this finding is not due to ethnic differences between patients and healthy individuals. While no one in the patient study group expressed the HLA-B*45 allele, 10 control group individuals, including 6 Caucasians, 1 African, 1 Amerindian, and 2 not identified, did express the HLA-B*45 allele.

The population of southern Brazil is heterogeneous in its racial composition and shows a high degree of miscegenation, consisting primarily of Caucasian descendants of Europeans, Africans, and Native Americans. According to the phenotypic classification system of HLA (Probst et al., 2000), the predominant population of the region selected for this study was of European origin (80.6\%), with a smaller contribution from Africans (12.5\%) and Native Americans $(7.0 \%)$. Considering this fact, our study also compared the results obtained for individuals of the patient study group and the control group for those who were reported to be Caucasian/white. The results for this comparison remained significant in implicating the HLA-C*04 allele $(P=0.0021$ and $P c=$ 0.0290 ) in the development of ACL (data not shown).

Petzl-Erler et al. (1991) also demonstrated the involvement of HLA class I in ACL in a different region of southern Brazil. These authors compared 43 subjects diagnosed with mucosal leishmaniasis to 111 healthy control individuals and found that detection of the HLA-B27 antigen $(P=0.029)$ was initially significant but not so after determination of the $P$ value. Our findings here corroborate the involvement of the HLA-B*27 allelic group in 15 patients who suffered from mucosal leishmaniasis $(P=0.0057$ and $P c=0.1578 ; O R=12.0994 ; 95 \% C l=1.7872-66.0541)$ (data not shown).

By analyzing the distribution of allele frequencies using the Arlequin 3.11 (http://cmpg. unibe.ch/software/arlequin3/), it was observed that in the ACL patient group, HLA-A $(P=0.0245)$ and HLA-C $(P=0.0311)$ had $P$ values $<0.05$, whereas we considered other alleles observed in the case group and all control groups in HWE $(P>0.05)$. Although there was a shift in HWE, the data obtained in this study support the potential role for specific HLA alleles in susceptibility or resistance to ACL. The association between $A C L$ and the $C$ locus and/or homozygosity may have influenced this deviation.

Some studies have shown an association of HLA class II with ACL (Petzl-Erler et al., 1991; Olivo-Díaz et al., 2004; Ribas-Silva et al., 2013). Considering this, future studies should also be performed using markers for HLA class II in southern Brazil.

We believe in the importance of developing new studies related to these and other genetic markers, investigating localized populations and analyzing allelic diversity, with the aim of identifying new associations or strengthening those that already exist. This knowledge will contribute to future prophylactic or therapeutic interventions in Brazilians at high risk for contracting American cutaneous leishmaniasis. 


\section{CONCLUSION}

These results implicate the involvement of the HLA class I gene in the susceptibility to and/or protection from ACL. Although we did not confirm the involvement of some of the alleles implicated, a significant association was observed between HLA-C*04 and ACL. In addition, this particular information was further supported by Bonferroni's correction, reinforcing the influence of this allele on ACL infection in the endemic regions of southern Brazil.

\section{REFERENCES}

Alves C, Vieira N, Meyer I, Oliveira-Alves C, et al. (2006). Antígenos de histocompatibilidade humanos e dermatologia: da pesquisa para a prática clínica. An. Bras. Dermatol. 81: 65-73.

Barbier D, Demenais F, Lefait JF, David B, et al. (1987). Susceptibility to human cutaneous leishmaniasis and HLA, Gm, Km markers. Tissue Antigens 30: 63-67.

BRASIL (2015). Ministério da Saúde. Casos de Leishmaniose Tegumentar Americana. Brasil, Grande Regiões e Unidades Federadas. 1990 a 2013. Available at [http://portalsaude.saude.gov.br/images/pdf/2014/setembro/09/LT-Casos.pdf]. Accessed Febuary 24, 2015.

Condino MLF, Galati EAB, Holcman MM, Salum MRB, et al. (2008). Leishmaniose tegumentar americana no Litoral Norte Paulista, período 1993 a 2005. Rev. Soc. Bras. Med. Trop. 41: 635-641.

Ellis SA and Ballingall KT (1999). Cattle MHC: evolution in action? Immunol. Rev. 167:159-168.

Lara ML, Layrisse Z, Scorza JV, Garcia E, et al. (1991). Immunogenetics of human american cutaneous leishmaniasis: Study of HLA haplotypes in 24 families from Venezuela. Hum. Immunol. 30: 129-135.

Lima AP, Minelli L, Teodoro U and Comunello E (2002). Distribuição da leishmaniose tegumentar por imagens de sensoriamento remoto orbital, no Estado do Paraná, Brasil. An. Bras. Dermatol. 77: 681-692.

Lonardoni MVC, Teodoro U, Arraes SMAA, Silveira TGV, et al. (1993). Nota sobre leishmaniose canina no noroeste do Estado do Paraná, sul do Brasil. Rev. Saude Publica 27:378-379.

Murray HW, Berman JD, Davies CR and Saravia NG (2005). Advances in leishmaniasis. Lancet 366: 1561-1577.

Olivo-Díaz A, Debaz H, Alaez C, Juárez-Islas V, et al. (2004). Role of HLA class II alleles in susceptibility to and protection from localized cutaneous leishmaniasis. Hum. Immunol. 65: 255-261.

Petzl-Erler ML, Belich MP and Queiroz-Telles F (1991). Association of mucosal leishmaniasis with HLA. Hum. Immunol. 32: 254-260.

Probst CM, Bompeixe EP, Pereira NF, et al (2000). HLA polymorphism and evaluation of European, African, and Amerindian contribution to the white and mulatto populations from Paraná, Brazil. Hum. Biol. 72: 597-617.

Rey L (2008). Parasitologia. 4th edn. Guanabara Koogan, Rio de Janeiro.

Romagnani S (1991). Human TH1 and TH2 subsets: doubt no more. Immunol. Today 12:256.

Rogers KA, DeKrey GK, Mbow ML, Gillespie RD, et al. (2002). Type 1 and type 2 responses to Leishmania major. FEMS Microbiol. Lett. 209: 1.

Ribas-Silva RC, Ribas AD, Santos MCG, Silva WV, et al. (2013). Association between HLA genes and american cutaneous leishmaniasis in endemic regions of Southern Brazil. BMC Infect. Dis.13: 198.

Sakthianandeswaren A, Foote SJ and Handman E (2009). The role of host genetics in leishmaniasis. Trends Parasitol. 25: 383-391.

Silveira TGV, Arraes SMAA, Bertolini DA, Teodoro U, et al. (1999). Observações sobre o diagnóstico laboratorial e a epidemiologia da leishmaniose tegumentar no Estado do Paraná, sul do Brasil. Rev. Soc. Bras. Med. Trop. 32: 413-423. 